\title{
Study of three-body charmonium decays at BABAR
}

\author{
Elisa Fioravanti* \\ INFN Ferrara, Via Saragat 1, 44122 Ferrara, Italy \\ E-mail: fioravanti@fe.infn.it
}

We report on new charmonium studies performed by the BABAR experiment located at the PEPII asymmetric energy $e^{+} e^{-}$collider at SLAC. We present analyses of the processes $\gamma \gamma \rightarrow K \bar{K} \pi$ in which we observe the decays $\eta_{c} \rightarrow K_{S} K^{ \pm} \pi^{\mp}$ and $\eta_{c} \rightarrow K^{+} K^{-} \pi^{0}$. We perform a Dalitz plot analysis of each $\eta_{c}$ final state, and extract the mass-dependent $I=1 / 2 K \pi \mathrm{S}$-wave amplitude and phase using a model-independent partial wave analysis approach. In addition, we study the reaction $e^{+} e^{-} \rightarrow \gamma_{I S R} J / \psi$, where $J / \psi \rightarrow \pi^{+} \pi^{-} \pi^{0}$, and $J / \psi \rightarrow K^{+} K^{-} \pi^{0}$, using events obtained from the Initial State Radiation (ISR) process. We measure the relative $J / \psi$ branching fraction, and perform a Dalitz plot analysis of each final state using an Isobar model and, for the $\pi^{+} \pi^{-} \pi^{0}$ final state, a Veneziano model.

XXIV International Workshop on Deep-Inelastic Scattering and Related Subjects

11-15 April, 2016

DESY Hamburg, Germany

\footnotetext{
${ }^{*}$ Speaker.
} 


\section{Charmonium Spectroscopy}

The charmonium spectrum consists of eight narrow states below the open charm threshold $\left(3.73 \mathrm{GeV} / \mathrm{c}^{2}\right)$, and several tens of states above the threshold. Below threshold almost all states are well-established. On the other hand, very little is known above threshold, where there are several new "Charmonium-like" states that are very difficult to accommodate in the charmonium spectrum.

B-factories are ideal places at which to study charmonium, since charmonium states are produced in four different processes:

- B decays: charmonium states of any quantum numbers can be produced;

- Two-photon production: in this process two virtual photons are emitted by the colliding $e^{+} e^{-}$ pair $\left(e^{+} e^{-} \rightarrow e^{+} e^{-} \gamma^{*} \gamma^{*} \rightarrow e^{+} e^{-}(c \bar{c})\right)$, and charmonium states with $J^{P C}=0^{ \pm+}, 2^{ \pm+}, 4^{ \pm+}, \ldots$ $3^{++}, 5^{++}, \ldots$ can be produced;

- Initial State Radiation (ISR): where a photon is emitted by the incoming electron or positron $\left(e^{+} e^{-} \rightarrow \gamma c \bar{c}\right)$, and only states with $J^{P C}=1^{--}$are formed;

- Double charmonium production: in this process a $J / \psi$ or a $\psi(2 S)$ is produced together with another charmonium state.

In this report we present two recent results:

- Measurement of the $I=1 / 2 K \pi S$-wave amplitude from Dalitz plot analyses of $\eta_{c} \rightarrow K \bar{K} \pi$ where the $\eta_{c}$ is produced in a two-photon interaction;

- Dalitz plot analyses of $J / \psi \rightarrow \pi^{+} \pi^{-} \pi^{0}$ and $J / \psi \rightarrow K^{+} K^{-} \pi^{0}$, where the $J / \psi$ is produced via $e^{+} e^{-}$annihilation after Initial-State Radiation.

\section{Measurement of the $I=1 / 2 K \pi$ S-wave amplitude from Dalitz plot analyses of $\eta_{c} \rightarrow K \bar{K} \pi$, where the $\eta_{c}$ is produced in a two-photon interaction}

We have performed a Dalitz plot analysis of $\eta_{c} \rightarrow K^{+} K^{-} \pi^{0}$, and of $\eta_{c} \rightarrow K^{+} K^{-} \eta$, in each case using an Isobar model [1]. The $K \bar{K} \pi$ systems are produced in two-photon interactions using an integrated luminosity of $519 \mathrm{fb}^{-1}$ recorded at center-of-mass energies at and near the $\Upsilon(n S)$ $(n=2,3,4)$ resonances. The analysis reported the first observation of $K_{0}^{*}(1430) \rightarrow K \eta$, and observed that $\eta_{c}$ decays to these final states are dominated by the presence of scalar meson resonances. We continued the study of $\eta_{c}$ decays to $K \bar{K} \pi$ and extracted the $I=1 / 2 K \pi$ S-wave by performing a Model Independent Partial Wave Analysis (MIPWA) of the $K_{s}^{0} K^{ \pm} \pi^{\mp}$ and $K^{+} K^{-} \pi^{0}$ final states [2].

The MIPWA Dalitz plot analyses of the $K_{s}^{0} K^{ \pm} \pi^{\mp}$ and $K^{+} K^{-} \pi^{0}$ final states were performed in the $\eta_{c}$ mass region using unbinned maximum likelihood fits. The results are summarized in Table 1 . The $K \pi \mathrm{S}$-wave amplitudes dominate, there is small $K_{2}^{*}(1430)^{0} \bar{K}$ contribution and there are also significant contributions from the $a_{0}(1950) \pi$ amplitude, where the $a_{0}$ is a new resonance which decays to $K \bar{K}$. A fit without this state gives a poor description of the data, as can be seen in Figure 1. The statistical significance for this state is $2.5 \sigma$ for $K_{s}^{0} K^{ \pm} \pi^{\mp}$ and $4.2 \sigma$ for $K^{+} K^{-} \pi^{0}$. The weighted averages for the parameter values of the $a_{0}$ are $m=(1931 \pm 14 \pm 22) \mathrm{MeV} / \mathrm{c}^{2}$ and $\Gamma=$ 


\section{$(271 \pm 22 \pm 29) \mathrm{MeV}$.}

In addition we performed an Isobar-model Dalitz plot analysis of the $K_{s}^{0} K^{ \pm} \pi^{\mp}$ and $K^{+} K^{-} \pi^{0}$ final states, in which all resonances were modeled using Breit-Wigner functions multiplied by the corresponding angular functions. The description of the data obtained using this model was significantly poorer than that obtained from the MIPWA method.

We compared these measurements of the $I=1 / 2 K \pi \mathrm{S}$-wave amplitude from $\eta_{c} \rightarrow K \bar{K} \pi$ with measurements from LASS [3], and from E791 [4]. While they observed similar phase behavior up to about $1.5 \mathrm{GeV} / \mathrm{c}^{2}$, they observed striking differences in the mass dependence of the amplitude.

Table 1: Results from the $\eta_{c} \rightarrow K_{s}^{0} K^{ \pm} \pi^{\mp}$ and $\eta_{c} \rightarrow K^{+} K^{-} \pi^{0}$ MIPWA.

\begin{tabular}{|c|c|c|c|c|}
\hline \multirow[b]{2}{*}{ Amplitude } & \multicolumn{2}{|c|}{$\eta_{c} \rightarrow K_{s}^{0} K^{ \pm} \pi^{\mp}$} & \multicolumn{2}{|c|}{$\eta_{c} \rightarrow K^{+} K^{-} \pi^{0}$} \\
\hline & Fraction (\%) & Phase (rad) & Fraction (\%) & Phase (rad) \\
\hline$\overline{(K \pi \text { S-wave }) \bar{K}}$ & $107.3 \pm 2.6 \pm 17.9$ & fixed & $125.5 \pm 2.4 \pm 4.2$ & fixed \\
\hline$a_{0}(980) \pi$ & $0.8 \pm 0.5 \pm 0.8$ & $1.08 \pm 0.18 \pm 0.18$ & $0.0 \pm 0.1 \pm 1.7$ & \\
\hline$a_{0}(1450) \pi$ & $0.7 \pm 0.2 \pm 1.4$ & $2.63 \pm 0.13 \pm 0.17$ & $1.2 \pm 0.4 \pm 0.7$ & $2.90 \pm 0.12 \pm 0.25$ \\
\hline$a_{0}(1950) \pi$ & $3.1 \pm 0.4 \pm 1.2$ & $-1.04 \pm 0.08 \pm 0.77$ & $4.4 \pm 0.8 \pm 0.8$ & $-1.45 \pm 0.08 \pm 0.27$ \\
\hline$a_{2}(1320) \pi$ & $0.2 \pm 0.1 \pm 0.1$ & $1.85 \pm 0.20 \pm 0.20$ & $0.6 \pm 0.2 \pm 0.3$ & $1.75 \pm 0.23 \pm 0.42$ \\
\hline$K_{2}^{*}(1430) \bar{K}$ & $4.7 \pm 0.9 \pm 1.4$ & $4.92 \pm 0.05 \pm 0.10$ & $3.0 \pm 0.8 \pm 4.4$ & $5.07 \pm 0.09 \pm 0.30$ \\
\hline Total & $116.8 \pm 2.8 \pm 18.1$ & & $134.8 \pm 2.7 \pm 6.4$ & \\
\hline$-2 \log \mathscr{L}$ & -4314.2 & & -2339 & \\
\hline$\chi^{2} / N_{\text {cells }}$ & $301 / 254=1.17$ & & $283.2 / 233=1.22$ & \\
\hline
\end{tabular}
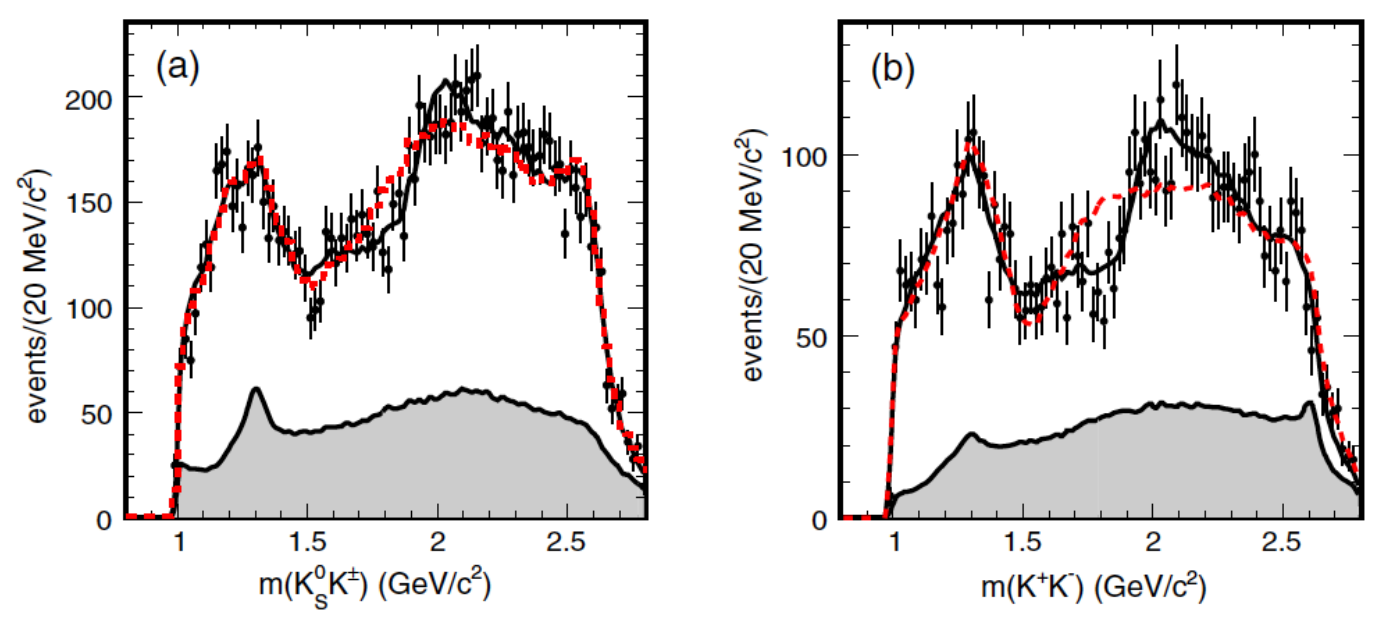

Figure 1: The mass projection for (a) $K_{s}^{0} K^{+}$from $\eta_{c} \rightarrow K_{s}^{0} K^{ \pm} \pi^{\mp}$, and b) $K^{+} K^{-}$from $\eta_{c} \rightarrow K^{+} K^{-} \pi^{0}$. The histograms show the MIPWA fit projection with (solid, black) and without (dashed, red) the presence of the $a_{0}(1950) \rightarrow K \bar{K}$ resonance. The shaded regions show the estimated background contributions obtained by interpolating the results of the Dalitz plot analyses of the sideband regions.

\section{Dalitz plot analysis of $J / \psi \rightarrow \pi^{+} \pi^{-} \pi^{0}$ and $J / \psi \rightarrow K^{+} K^{-} \pi^{0}$ produced via $e^{+} e^{-}$ annihilation after Initial-State Radiation}

We studied $J / \psi \rightarrow \pi^{+} \pi^{-} \pi^{0}$ and $J / \psi \rightarrow K^{+} K^{-} \pi^{0}$ decays, where the $J / \psi$ was produced in 

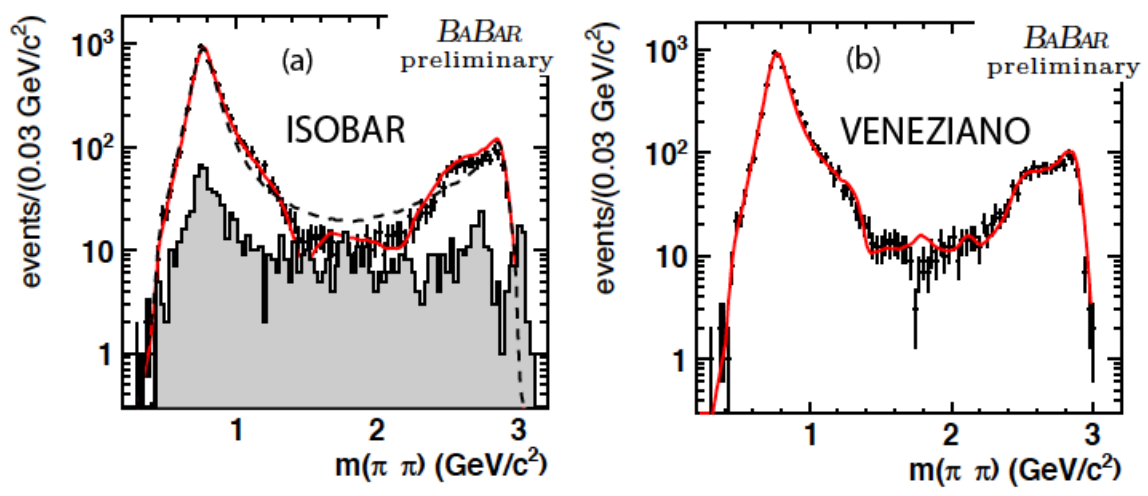

Figure 2: The $\pi \pi$ mass projections for $J / \psi \rightarrow \pi^{+} \pi^{-} \pi^{0}$. The fit in (a) makes use of the Isobar model and the shaded histogram represents the background distribution obtained from the $J / \psi$ sidebands. The dashed curve in (a) is the result of the fit with only the $\rho(770)$ resonance. The fit it (b) makes use of the Veneziano model.

Table 2: Results from the Dalitz-plot analysis of the $J / \psi \rightarrow \pi^{+} \pi^{-} \pi^{0}$ channel.

\begin{tabular}{lrr|r}
\hline Final state & Isobar Fraction $(\%)$ & Phase (radians) & Veneziano Fraction $(\%)$ \\
\hline$\rho(770) \pi$ & $119.0 \pm 1.1 \pm 3.3$ & 0. & $120.0 \pm 1.9$ \\
$\rho(1460) \pi$ & $16.9 \pm 2.0 \pm 3.1$ & $3.92 \pm 0.05 \pm 0.11$ & $1.53 \pm 0.13$ \\
$\rho(1700) \pi$ & $0.1 \pm 0.1 \pm 0.2$ & $1.01 \pm 0.35 \pm 0.79$ & $0.84 \pm 0.08$ \\
$\rho(2150) \pi$ & $0.04 \pm 0.05 \pm 0.02$ & $1.89 \pm 0.30 \pm 0.48$ & $2.03 \pm 0.17$ \\
$\rho_{3}(1690) \pi$ & $136.0 \pm 2.3 \pm 4.3$ & & $0.09 \pm 0.02$ \\
Sum & $764 / 552$ & & $124.5 \pm 2.3$ \\
$\chi^{2} / v$ & & & $780 / 554$ \\
\hline
\end{tabular}

Table 3: Results from the Dalitz-plot analysis of the $J / \psi \rightarrow K^{+} K^{-} \pi^{0}$ channel.

\begin{tabular}{lrr}
\hline Final state & Fraction $(\%)$ & Phase (radians) \\
\hline$K^{*}(892) K$ & $87.8 \pm 2.0 \pm 1.7$ & 0. \\
$\rho(1450)^{0} \pi^{0}$ & $11.5 \pm 2.1 \pm 2.1$ & $-2.81 \pm 0.25 \pm 0.36$ \\
$K^{*}(1410) K$ & $1.7 \pm 0.7 \pm 1.1$ & $2.89 \pm 0.35 \pm 0.08$ \\
$K_{2}^{*}(1430) K$ & $3.8 \pm 1.4 \pm 0.5$ & $-2.42 \pm 0.22 \pm 0.07$ \\
$\rho(1700)^{0} \pi^{0}$ & $0.9 \pm 1.0 \pm 0.6$ & $1.06 \pm 0.20 \pm 0.7$ \\
Total & $105.6 \pm 3.4 \pm 3.0$ & \\
$\chi^{2} / v$ & $94 / 92$ & \\
\hline
\end{tabular}

$e^{+} e^{-}$annihilation after initial-state radiation, using an integrated luminosity of $519 \mathrm{fb}^{-1}$ recorded at center-of-mass energies at and near the $\Upsilon(n S)(n=2,3,4)$ resonances. Only an unpublished result exists for a Dalitz plot analysis of $J / \psi$ decay to $\pi^{+} \pi^{-} \pi^{0}$ [5].

While large samples of $J / \psi$ decays exist, some branching fractions remain poorly measured. In particular the $J / \psi \rightarrow K^{+} K^{-} \pi^{0}$ branching fraction has been measured by MarkII [6] using only 
25 events. The BESII experiment [7] has performed an angular analysis of $J / \psi \rightarrow K^{+} K^{-} \pi^{0}$, which required the presence of a broad $J^{P C}=1^{--}$state in the $K^{+} K^{-}$threshold region; this was interpreted as being due to a multi-quark state.

We have performed Dalitz-plot analyses of $J / \psi \rightarrow \pi^{+} \pi^{-} \pi^{0}$ and $J / \psi \rightarrow K^{+} K^{-} \pi^{0}$ decays using unbinned maximum likelihood fits. For the first $J / \psi$ decay mode, we used both an Isobar and a Veneziano model, while for the second $J / \psi$ decay mode, only an Isobar model has been used, due to limited statistics.

In Table 2 we summarize the results from the Dalitz-plot analysis of the $J / \psi \rightarrow \pi^{+} \pi^{-} \pi^{0}$ channel. The decay is dominated by $\rho(770) \pi$ amplitudes. Using an Isobar model, there is also an important contribution from the $\rho(1450) \pi$ amplitude. From the fit the mass $m(\rho(1450))=(1361$ $\pm 43) \mathrm{MeV} / \mathrm{c}^{2}$, and width $\Gamma(\rho(1450))=(479 \pm 63 \mathrm{MeV})$ values were extracted, and these are consistent with the PDG [8] averages. The Isobar and Veneziano models give similar data representation, but they require different fractional contributions. The projections of the fits on the $\pi \pi$ mass distributions are shown in Figure 2.

In Table 3 we summarize the results from the Dalitz-plot analysis of the $J / \psi \rightarrow K^{+} K^{-} \pi^{0}$ channel. For this channel, only the Isobar model has been used due to limited statistics. The decay is dominated by $K^{*}(892) \bar{K}$ amplitudes. There is also an important contribution from the $\rho(1450) \pi$ amplitude. In the fit the mass and the width of the $\rho(1450)$ were fixed to the values obtained from the $\pi^{+} \pi^{-} \pi^{0}$ Isobar analysis.

Using the results for these $J / \psi$ decay modes obtained with the Isobar model, we measure the branching ratio $\mathscr{R}(\rho(1450))=\frac{\mathscr{B}\left(\rho(1450) \rightarrow K^{+} K^{-}\right)}{\mathscr{B}\left(\rho(1450) \rightarrow \pi^{+} \pi^{-}\right)}=0.190 \pm 0.042 \pm 0.049$.

\section{References}

[1] B. Aubert et al. (BABAR Collaboration), Phys. Rev. D 81, 092003 (2010).

[2] J. P. Less et al. (BABAR Collaboration), Phys. Rev. D 93, 012005 (2016).

[3] D. Aston et al. (LASS Collaboration), Nucl. Phys. B 296, 493 (1988).

[4] E. M. Aitala et al. (E791 Collaboration), Phys. Rev. D 73, 032004 (2006); Phys Rev. D 74, 059901 (E) (2006).

[5] L. P. Chen and W. Dunwoodie, (MarkIII Collaboration), SLAC-PUB-5674, (1991).

[6] M.E.B. Franklin et al. Phys. Rev. Lett. 51, 963 (1983).

[7] M. Ablikim et al. (BESII Collaboration), Phys. Rev. Lett. 97, 142002 (2006).

[8] K.A. Olive et al. (Particle Data Group), Chin. Phys. C 38, 090001 (2014). 